\title{
A NOTE ON GENERALIZED HYBRID TRIBONACCI NUMBERS
}

\author{
TÜLAY YAĞMUR \\ Department of Mathematics \\ Aksaray University \\ 68100 Aksaray, Turkey
}

e-mail: tulayyagmurr@gmail.com / tulayyagmur@aksaray.edu.tr

\begin{abstract}
In this paper, we introduce the generalized hybrid Tribonacci numbers. These numbers can be considered as a generalization of the generalized complex Tribonacci, generalized hyperbolic Tribonacci and generalized dual Tribonacci numbers. We also obtain some identities for these numbers.

Keywords: complex numbers, hyperbolic numbers, dual numbers, hybrid numbers, generalized Tribonacci numbers.

2010 Mathematics Subject Classification: Primary: 11B37; Secondary: 11B39, 97F50.
\end{abstract}

\section{REFERENCES}

[1] T. Andreescu and D. Andrica, Complex Numbers from A to ... Z (Birkhauser/ Springer, New York, 2014).

[2] P. Catarino, On k-Pell hybrid numbers, J. Discrete Math. Sci. and Cryptography 22 (2019) 83-89. doi:10.1080/09720529.2019.1569822

[3] F. Catoni, R. Cannata, V. Catoni and P. Zampetti, Hyperbolic trigonometry in twodimensional space-time geometry, IL Nuovo Cimento B 118 (2003) 475-492. doi:10.1393/ncb/i2003-10012-9

[4] F. Catoni, D. Boccaletti, R. Cannata, V. Catoni, E. Nichelatti and P. Zampetti, The Mathematics of Minkowski Space-Time: With an Introduction to Commutative Hypercomplex Numbers (Birkhauser, Basel, 2008).

[5] G. Cerda-Morales, On a Generalization for Tribonacci Quaternions, Mediterr. J. Math. 14:239 (2017) 1-12.

doi:10.1007/s00009-017-1042-3 
[6] G. Cerda-Morales, Investigation of Generalized Hybrid Fibonacci Numbers and Their Properties, arXiv preprint, arXiv: 1806.02231v1 (2018).

[7] F.M. Dimentberg, The method of screws and calculus of screws applied to the theory of three dimensional mechanisms, Adv. Mech. 1 (1978) 91-106.

[8] I. Fischer, Dual-Numbers Methods in Kinematics, Statics and Dynamics (CRC Press, Boca Raton-London-New York-Washington D.C., 1998).

[9] N.A. Gromov and V.V. Kuratov, All possible Cayley-Klein contractions of quantum orthogonal groups, Yadernaya Fizika 68 (2005) 1752-1762.

[10] N.A. Gromov and V.V. Kuratov, Possible quantum kinematics, J. Math. Phys. 47 (2006) 013502-013502-9. doi:10.1063/1.2157093

[11] N.A. Gromov, Possible quantum kinematics. II. Nonminimal case, J. Math. Phys. 51 (2010) 083515-083515-12. doi:10.1063/1.3460841

[12] D. Hestenes, Vectors, Spinors and Complex Numbers in Classical and Quantum Physics, Amer. J. Phys. 39 (1971) 1013-1027. doi:10.1119/1.1986363

[13] V.V. Kisil, Geometry of Mobius Transformations: Elliptic, Parabolic and Hyperbolic Actions of SL2(R) (Imperial College Press, London, 2012).

[14] C. Kizilates, P. Catarino and N. Tuglu, On the Bicomplex Generalized Tribonacci Quaternions, Mathematics 7, 80 (2019). doi:10.3390/math7010080

[15] D.P. Mandic and V.S. Lee Goh, The Magic of Complex Numbers: Complex Valued Nonlinear Adaptive Filters (John Wiley and Sons, Hoboken, New Jersey, 2009).

[16] M. Özdemir, Introduction to Hybrid Numbers, Adv. Appl. Clifford Algebras 28 (11) 2018. doi:10.1007/s00006-018-0833-3

[17] S. Pethe, Some Identities for Tribonacci Sequences, The Fibonacci Quarterly 26 (1988) 144-151.

[18] J. Rooney, Generalized Complex Numbers in Mechanics, Adv. Theory Pract. Robots Manip. Mech. Machine Sci. 22 (2014) 55-62.

[19] A.G. Shannon and A.F. Horadam, Some Properties of Third-Order Recurrence Relations, The Fibonacci Quarterly 10 (1972) 135-145.

[20] A. Szynal-Liana, The Horadam Hybrid Numbers, Discuss. Math. Gen. Alg. and Appl. 38 (2018) 91-98. doi:10.7151/dmgaa.1287

[21] A. Szynal-Liana and I. Wloch, On Jacobsthal and Jacobsthal-Lucas Hybrid Numbers, Ann. Math. Silesianae 33 (2019) 276-283. doi:10.2478/amsil-2018-0009 
[22] W.B. Vasantha Kandasamy and F. Smarandache, Dual Numbers (Zip Publishing, Ohio, 2012).

[23] G.R. Veldkamp, On the use of dual numbers, vectors and matrices in instantaneous, spatial kinematics, Mech. Mach. Theory 11 (1976) 141-156. doi:10.1016/0094-114X(76)90006-9

[24] I.M. Yaglom, Complex Numbers in Geometry (Academic Press, New York, 1968).

[25] I.M. Yaglom, A Simple Non-Euclidean Geometry and Its Physical Basis (SpringerVerlag, New York, 1979).

[26] C.C. Yalavigi, Properties of Tribonacci numbers, The Fibonacci Quarterly 10 (1972) 231-246.

Received 2 July 2019

Revised 30 January 2020

Accepted 7 June 2020 